\title{
APOFDM system design based on all phase FFT technology
}

\author{
Mengjiao $\mathbf{W u}^{\mathrm{a}}$, Jun Yang ${ }^{\mathbf{b}^{*}}$ \\ School of Information Science and Engineering, Yunnan university, Kunming, China \\ a344786838@qq.com, b*junyang@ynu.edu.cn
}

Keywords: OFDM system; FFT; APOFDM system; MATLAB

\begin{abstract}
The traditional OFDM system as an important part of current wireless communication technology, its advantages are obvious, but one of shortcoming is easily affected by the frequency deviation. An effective solution is to improve data signal processing technology in the front of the system, thus can reduce the effects of system faults and improve the performance of the system. All phase data processing under the research idea of it is in this, after 20 years of research and development, now all phase FFT technology has been applied to image processing, signal processing, signal spectrum analysis, wireless communication transmission, etc. In this paper, using all phase FFT processing technology and detailed the APOFDM, Finally, using MATLAB simulation tools to analyze its advantages.
\end{abstract}

\section{Introduction}

For the traditional OFDM system,the generation of orthogonal signal, sending and receiving is the key to the whole system [1].In mathematics can be achieved by discrete fourier transform (DFT) and the inverse transform (IDFT) processing these signals, In practice, to facilitate the implementation of the hardware and improve the speed of processing,can use the fast Fourier transform (FFT) and inverse transformation instead of (IFFT) implementation[2].

\section{The theory of APOFDM system}

The conventional OFDM system has only $\mathrm{N}$ data signals for the data sequence, and for the APOFDM system, it is necessary to preprocess the $2 \mathrm{~N}-1$ data signal points in one symbol period[3]. So the difference between the two is mainly in the system's sender, the IFFT transform $\mathrm{N}$ data signal output to repeat, resulting in $2 \mathrm{~N}-1$ data signal point.Corresponding to the receiver, the signal is decoded with full-phase FFT technology instead of the traditional FFT processing technology after Serial parallel conversion. Other structures are basically similar.

Assuming that the N-channel binary data of the sender is denoted as $X=\left[X_{0}, X_{1}, \ldots, X_{N-1}\right]$,copied to the $2 \mathrm{~N}$-channel signal $\mathrm{X}$ ', After modulation mapping and the pilot information is inserted, The data into the length of the $2 \mathrm{~N}$ point of the IFFT module is converted to the time domain signal vector can be expressed as:

$$
x(n)=\operatorname{IFFT}\left(X^{\prime}\right)=\left[X_{0}, X_{1}, \ldots, X_{N-1}, X_{0}, X_{1}, \ldots, X_{N-1}\right]
$$

Where $0 \leq n \leq 2 N-1$, we can insert the " 0 " value of the pilot symbol in the signal. According to the FFT property, we can see that the formula (1) is a cycle of length N. and then through the addition of NG cyclic prefix, with NG is greater than the channel impulse response, the impulse response and transmission time domain data sequence linear convolution into a round Product, tTo a certain extent, it can counteract the effect of intersymbol interference, and finally generate the transmitted symbols by the Serial parallel conversion and filtering[4]. 
At the receiver of the system, the serial symbols are sampled and converted into parallel samples, and then the cyclic prefix is removed. At this time for the ideal with the channel, the signal distortion mainly from the frequency offset and additive white Gaussian noise between the transmission and receiver. The corresponding $2 \mathrm{~N}$ channel received signal vector is:

$$
x^{\prime}(n)=x(n) e^{j n \Delta f}+w(n) \quad 0 \leq n \leq 2 N-1
$$

Where $\mathrm{f}$ is the OFDM subcarrier frequency interval, and $\mathrm{w}(\mathrm{n})$ represents the additive Gaussian white noise with mean 0 and variance d.remove the value of $x^{\prime}(0)$, and $x^{\prime}(N)$ as the center of the cycle to flip to $2 \mathrm{~N}-1$ items, each row superimposed normalized, and then $\mathrm{x}^{\prime}(\mathrm{N})$ as a starting point to cut off processing to generate full-phase FFT Signal vector:

$$
y(n)=\frac{1}{N}\left[(2 N-n) x^{\prime}(n)+(n-N) x^{\prime}(n-N)\right]
$$

In the above equation, $N \leq n \leq 2 N-1$,mathematically because of $n(2 N-n)+(n-N)(n-N)]=N^{2} \quad$, it is proved that the generated full-phase FFT signal is independent of the position of the sampling point. The significance is that the full-phase FFT vector generated during the signal transmission eliminates the relative frequency offset generated by the system, which is one of the advantages that the system introduces in full-phase FFT technology[5].

Then do y (n) do N point of the FFT operation, the receiver data can be expressed as:

$$
Y(k)=|X(\mathrm{k})|^{2} e^{j \varphi_{0}}+W(k)
$$

\section{All phase FFT technique}

APOFDM system is the main difference with the traditional OFDM system is in the process of transmission of signal modulation and demodulation joined the all phase FFT technology, the technology is in order to solve the problem of on truncation, causes the signal data in frequency spectrum, phase, etc and all kinds of distortion. OFDM sub-carrier signal modulation and demodulation process mainly USES the IFFT/FFT transform of digital signal processing, in the process, in order to keep the carrier orthogonality and reduce the effects of frequency deviation, joining the concept of all phase FFT technique.

\section{The MATLAB simulation}

In the signal preprocessing is completed, in order to get the results of all phase FFT and general FFT processing operation. For OFDM the multicarrier signal, can be combined with the simulation tool MATLAB verifies all phase FFT technique are introduced to its advantages.

Now suppose multiple frequency sampling time domain signal of $\mathrm{x}(\mathrm{n})$ for the two frequency sine wave superposition, plus noise, as shown in figure 1.

On FFT and all phase after FFT processing respectively, as shown in figure 2 and 3, respectively to compare their amplitude spectrum and phase spectrum, from the figure, the traditional FFT amplitude spectrum is relatively flat, the peak approximately $60 \mathrm{db}$, frequency on both sides of the tail is larger, the phase spectrum is not equal to signal in the early phase, the frequency $\mathrm{f}=50 \mathrm{hz}, \mathrm{f}=$ $100 \mathrm{hz}$ in the main line phase and on the side of the line phase and jump phenomenon occurs, and after all phase FFT processing, signal amplitude spectrum was steep, peak of about $110 \mathrm{db}$, equal to 2 times the peak of the traditional FFT, frequency on both sides of the tail is smaller, the phase spectrum is continuous, because of the reduced frequency spectrum leakage, the intercarrier interference caused by the carrier frequency offset sensitivity is lower. The simulation results with the previous section mathematically proved to be consistent, to solve the traditional OFDM system signals the influence of frequency offset and phase noise faults provides the improvement program. 


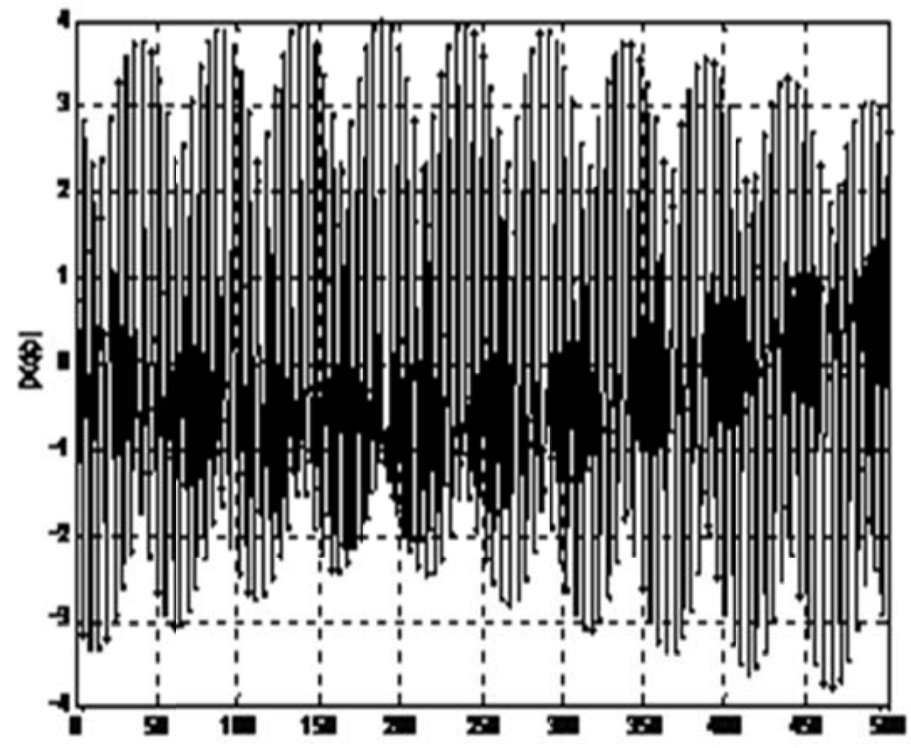

Fig.1 sampling signal waveform figure
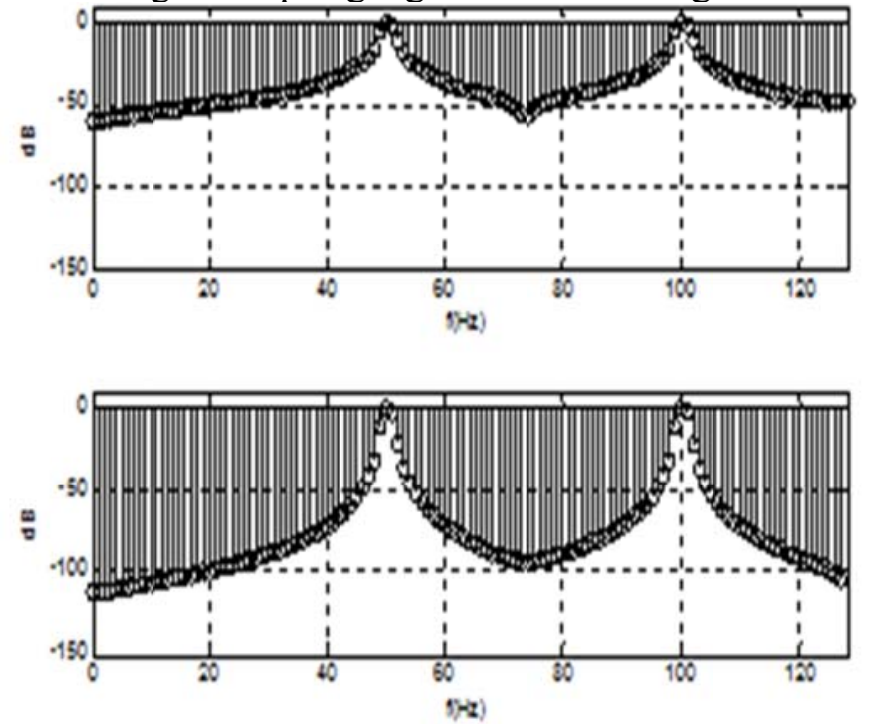

Fig. 2 signal FFT processing and all phase FFT processing amplitude spectrum comparison chart
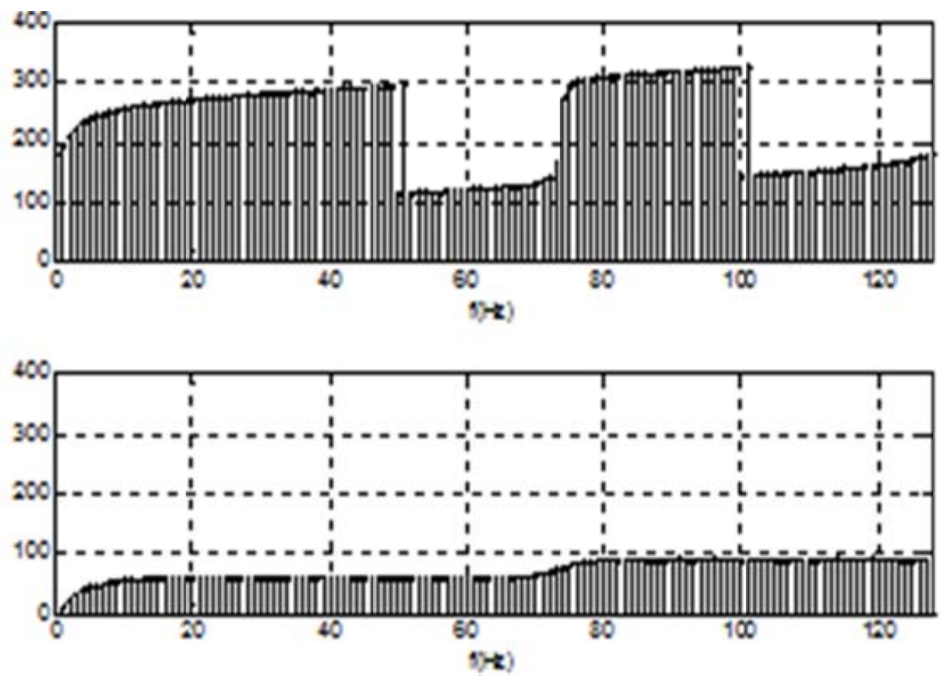

Fig. 3 signal FFT processing and all phase FFT processing phase spectrum comparison chart 


\section{Summary}

This paper briefly introduces the basic principle of OFDM system, due to one of its weaknesses is easily affected by the frequency deviation, puts forward the all phase FFT technology instead of FFT technology APOFDM system overall design, finally take advantage of MATLAB simulation tool for analysis.

\section{References}

[1] Zhou Bing, liu, from the side. Based on pilot channel estimation method in OFDM system research [J]. Journal of China institute of electronics, 2011, 8 (4) : 397-400.

[2] Amy polumbo etropulu, R.Z hang and R.L in, Blind OFDM channel estimation through simple linear precoding [J]. IEEE Trans. On Wireless Communication, vol. 3, no. 2, pp. 647-655, March 2004

[3] Sun Cuizhen Ceng Zhaohua. An improved fast fading OFDM channel estimation algorithm [J]. Journal of xi 'an university of science and technology. 2008, 3 (1) : 128-131.

[4] N.M.I drees, W.H aselmayr, D.S chellander A.S pringer, Time variant channel estimation using a modified complex an exponential basis expansion model in LTE - OFDM systems [J]. IEEE 21 st International Symposium on Personal Indoor and Mobile Radio Communications, 2010:603-607.

[5] ZhangHuan Ye Fang, yi-bing li. Channel estimation algorithms for OFDM systems design based on FPGA [J]. Journal of information technology. (6) : 2009-28 33. 